\title{
Wideband Josephson THz flux-flow oscillator integrated with the slot lens antenna and the harmonic mixer
}

\author{
$\underline{\text { N.V.Kinev }}^{1}$, K.I. Rudakov ${ }^{1,2,3}$, L.V. Filippenko ${ }^{1}$, A.M. Baryshev ${ }^{3}$, V.P. Koshelets ${ }^{1}$ \\ ${ }^{1}$ Kotel'nikov Institute of Radio Engineering and Electronics of RAS, 125009 Moscow, Russia, nickolay@hitech.cplire.ru \\ ${ }^{2}$ Moscow Institute of Physics and Technology, 141701 Dolgoprudny, Russia \\ ${ }^{3}$ University of Groningen, 9712 CP Groningen, Netherlands
}

\section{Introduction}

Terahertz (THz) wideband sources are required nowdays in many field, such as spectroscopy, astronomy and radio physics. We present a new implementation for a flux-flow oscillator (FFO) based on a long Josephson junction providing the $\mathrm{THz}$ emission in the open space. The FFO was used earlier in our team merely as an on-chip heterodyne for the SIS receiver [1-2], it has a really wide operating range up to $100 \%$ of central frequency and a power of about $1 \mu \mathrm{W}$. Based on the $\mathrm{Nb} / \mathrm{AlN} / \mathrm{NbN}$ trilayer the FFO with dimensions $400 \times 16 \mu \mathrm{m}^{2}$ provides the output radiation from $200 \mathrm{GHz}$ up to $750 \mathrm{GHz}$ with the spectral linewidth of about $1 \mathrm{MHz}$. The upper operating frequency can reach $1 \mathrm{THz}$. The phase-lock loop (PLL) is used for the phase locking of the emission collecting up to $97 \%$ of the output $\mathrm{THz}$ power in the peak with a width of about $40 \mathrm{kHz}$. Such oscillator implemented as an external source could be useful for many tasks gas spectroscopy, heterodyne receivers, etc.

\section{Concept of the THz oscillator \& numerical simulations}

The principle of the FFO operation is discussed elsewhere [3-4]. The operating frequency is defined strictly by the Josephson equation

$$
\mathrm{h} f=2 \mathrm{e} V \text {, }
$$

where $\mathrm{V}$ is the $\mathrm{DC}$ voltage on the junction. The original idea of this work is the integration of the FFO with a harmonic mixer (HM) for the feedback locking loop and a transmitting slot antenna on a single chip (fig. 1a) placed on the back surface of the elliptical lens (fig.1b). The chip substrate and the lens are both made of silicon. Thus, the main task is the coupling of the oscillator having low output impedance (less than $1 \Omega$ ) to the lens antenna having high impedance (tens of $\Omega$ ) and forming a beam pattern required for applications, and simultaneous coupling to the harmonic mixer based on SIS junction having the area about $1 \mu \mathrm{m}^{2}$ and the impedance of about several $\Omega$. Coupling to the antenna should be as high as possible, while coupling to the HM should be just enough for properly PLL operation and not take away much power (commonly 10-20\%). Both couplings with the antenna and the HM should be in the same frequency range, which is required to be as wide as possible. The slot antenna is fabricated of superconducting $\mathrm{Nb}$ thin film, as well as the transmitting microstrip lines between the FFO, antenna and HM (the thickness is about $200-450 \mathrm{~nm}$ ). The chip with the planar integrated circuit mounted on the lens is placed in the liquid helium cryogenic system with the temperature $4.2 \mathrm{~K}$.

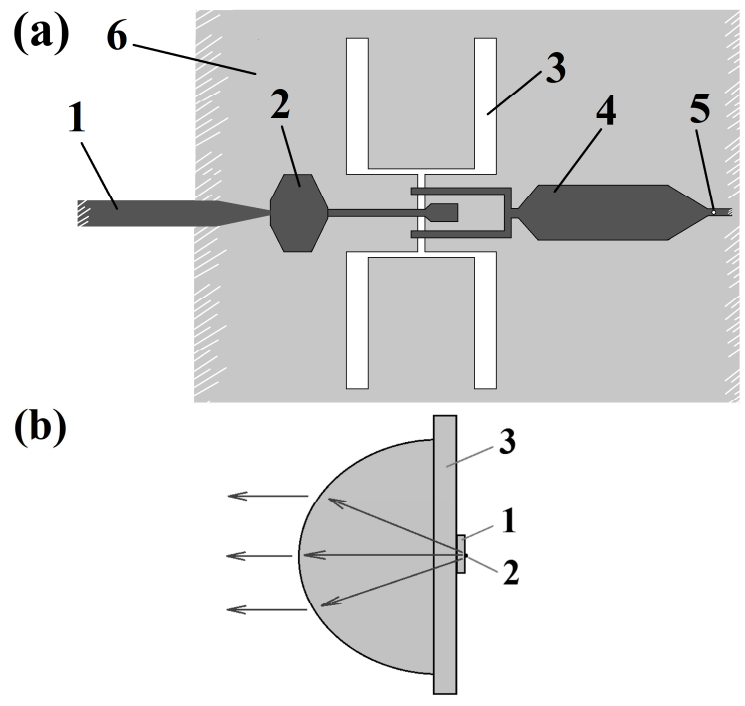

Fig. 1. (a) layout of the planar structure of $\mathrm{THz}$ oscillator (1) based on a long SIS junction coupled by microstrip line (2) to slot antenna (3) and by microstrip line (4) to harmonic SIS mixer (5), metallization layer (6) of antenna is also the bottom electrode of the microstrip and the SIS junctions;

(b) scheme of the chip (1) with oscillator and antenna shown on (a) placed at the far focus (2) of the silicon lens (3). The scheme (b) is not to scale.

The main calculations for the designs of the oscillator coupled to the antenna and HM are made by using the specialized software for microwave 3D modeling. Three designs of the "oscillator \& antenna structure" were developed for the central frequencies $350 \mathrm{GHz}, 450 \mathrm{GHz}$ and $600 \mathrm{GHz}$, hereinafter called $\mathrm{A}-350, \mathrm{~A}-450$ and $\mathrm{A}-600$ respectively. More than $70 \%$ of oscillator output power is radiated in the ranges of $250-410 \mathrm{GHz}, 330-570 \mathrm{GHz}$ and $420-$ $700 \mathrm{GHz}$ for three designs, the calculation results for the emitted power vs frequency will be shown together with the experimental results in the next section. Designs A-450 and A-600 contains all the elements shown in fig.1a, but A-350 doesn't contain the HM with a corresponding coupling line and has no ability to lock the oscillator. A-350 design will be upgraded in the next step of this work. In the fig. 2 the calculated beam patterns at the fixed frequency for $350 \mathrm{GHz}$, $450 \mathrm{GHz}$ and $600 \mathrm{GHz}$ antenna designs are shown, the main power is concentrated in the center lobe.

\section{Experimental results: preliminary testing}

The batch of experimental samples based on $\mathrm{Nb} / \mathrm{AlN} / \mathrm{NbN}$ was fabricated according to the developed designs. The current density of the SIS trilayes on the batch is about $j_{c}=10 \mathrm{kA} / \mathrm{cm}^{2}$, this corresponds to parameter $R_{n} S$ of about $20 \Omega \cdot \mu \mathrm{m}^{2}$. The quality fac- 
tor of the junctions defined as the ratio of "sub-gap" resistance to normal resistance $R_{j} / R_{n}$ is about 30 .

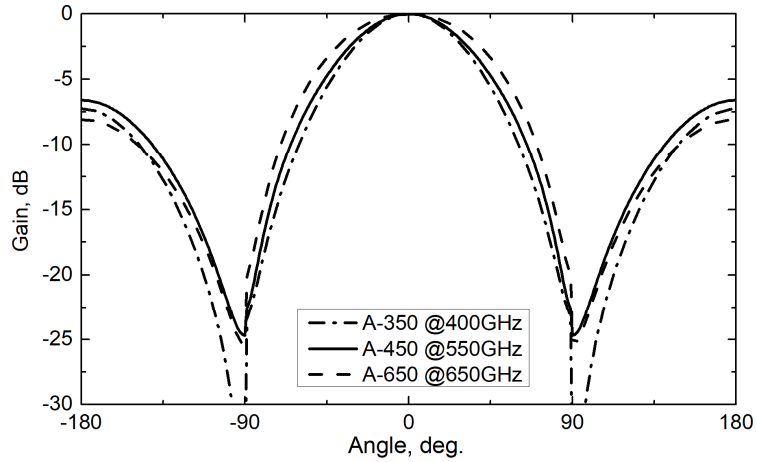

Fig. 2. Numerically simulated beam patterns of three antenna designs at the fixed frequency in the region of operation.

The pumping of the SIS harmonic mixer by FFO power leads to the appearance of current quasiparticle step on the IVC which is measured directly in the whole frequency range of the FFO operation. In the fig. 3 the experimental results for frequency dependences of HM pumping are presented together with the calculation results of the absorbed power for the designs A-450 and A-600. There is a good agreement of the experiment with numerical simulations.

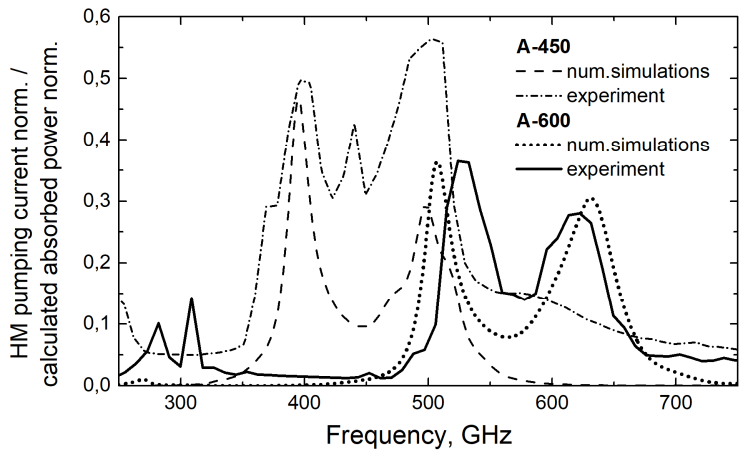

Fig. 3. Experimental results of HM pumping by FFO power and calculation results of the absorbed FFO power by HM. The HM pumping current is normalized to the "current jump" at the gap SIS voltage. The absorbed power is normalized to the total output FFO power.

For the measurements of the FFO emission in the open space by lens antenna the superconducting integrated spectrometer (SISP) is used [5]. Two liquid helium cryostats for the oscillator and the receiver were used and set opposite each other, so that the quasioptical windows of the cryostats were oriented toward one another. The local oscillator of $19-21 \mathrm{GHz}$ is used for the mixing by the HM of the FFO signal and the $n$-th harmonics of the oscillator. Additionally, the PLL system is used for locking the signal. The radiated spectral line could be measured simultaneously by the $\mathrm{HM}$ in the intermediate frequency (IF) range $0-800 \mathrm{MHz}$ and by the spectrometer in the IF range 4 - $8 \mathrm{GHz}$. The emission in the open space was successfully obtained in the wide region at some frequency points of operation and studied by the SISP as well as by the HM and the feedback loop. The results of the first measurements together with the transmitting antenna characteristics are shown in the fig.4. The spectral lines emitted by FFO have the Lorenzian shape with a linewidth of about 2-15 MHz; the ratio of signal (spectral line) power to noise power is shown on the right axis in fig.4. Numerical simulations for A-350 $(250-410 \mathrm{GHz}$ range) are not presented since an experiment still was not carried out at $\mathrm{THz}$ frequencies for this design. Such experiment requires another type of receiver (a Golay cell or a $\mathrm{Si}$ bolometer).

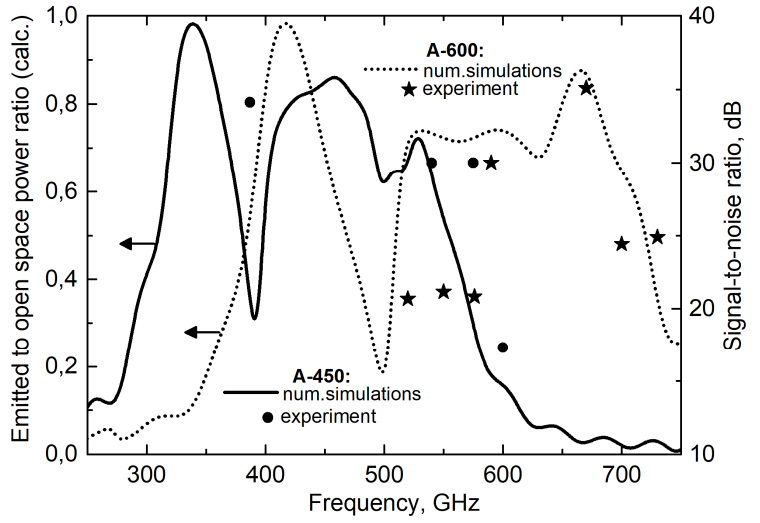

Fig. 4. Calculation results of power emitted by antenna to open space normalized to the total output FFO power (left axis); experimental points of output emission detected by $\mathrm{SISP}$ at some frequencies (right axis).

\section{Conclusion}

The superconducting $\mathrm{THz}$ oscillator based on the long Josephson junction with unidirectional flow of the fluxons (a flux-flow oscillator or a FFO) is an encouraging solution of the $\mathrm{THz}$ source for the tasks where wideband frequency tuning is required and the high power is not necessary. We proposed the idea and implementation for the external $\mathrm{THz}$ source based on the FFO integrated with the harmonic mixer and the transmitting slot antenna integrated on a single chip with the oscillator. The lens is used forming the narrow beam pattern. Three antenna designs that covers the $0.25-0.7 \mathrm{THz}$ region are developed and numerically simulated, two designs for $0.33-0.57 \mathrm{THz}$ and $0.42-0.7 \mathrm{THz}$ are experimentally studied. The emission to open space is measured by external high resolution spectrometer.

This work is supported by Russian Science Foundation (project № 17-79-20343).

\section{References}

1. Koshelets, V.P., Shitov, S.V. Integrated Superconducting Receivers // Supercond. Sci. Technol. 2000. V. 13. P. R53-R69.

2. Lange, G., Boersma, D., Dercksen, J. et al. Development and Characterization of the Superconducting Integrated Receiver Channel of the TELIS Atmospheric Sounder// Supercond. Sci. Technol. 2010. V. 23. 045016 (8pp).

3. Nagatsuma, T., Enpuku, K., Irie, F., Yoshida, K. Flux-flow type Josephson oscillator for millimeter and submillimeter wave region // J. Appl. Phys. 1983. V. 54 P. 3302-3309.

4. Zhang, $Y$. Theoretical and experimental studies of the flux-flow type Josephson oscillator. 1991. Gothenburg: Chalmers University of Technology.

5. Koshelets, V.P., Dmitriev, P.N. et al. Superconducting Integrated Terahertz Spectrometers. // IEEE Trans. Terahertz Sci. Technol. 2015. Vol. 5, No. 4. P. 687-694. 